\title{
Chorioretinitis Sclopetaria: A Case Report
}

\author{
Vadim Igal $^{a, b} \quad$ Yael Sara Pikkel Igal ${ }^{a} \quad$ Yoav Yechezkel Pikkel ${ }^{c}$ \\ ${ }^{a}$ Faculty of Medicine, Bar-Ilan University, Safed, Israel; ${ }^{b}$ Department of Ophthalmology, \\ Ziv Medical Center, Safed, Israel; ${ }^{\circ}$ Carmel Medical Center, Haifa, Israel
}

\section{Keywords}

Chorioretinitis sclopetaria $\cdot$ Shrapnel trauma

\begin{abstract}
We present the case of a 43-year-old male with a war injury that manifested in damage to the retina in his right eye causing chorioretinitis sclopetaria. Chorioretinitis sclopetaria is a characteristic pattern of choroidal and retinal damage that is caused by a high-velocity projectile passing into the orbit, in close proximity to the eye globe. We discuss this rare situation, the injury mechanism, suggested treatment, and prognosis.

(C) 2017 The Author(s)

Published by S. Karger AG, Basel
\end{abstract}

\section{Introduction}

Sclopetaria is a secondary outcome of a decelerating object passing at a high velocity adjacent to the sclera [1]. While passing close to the globe, after shock forces are generated, rupturing of the choroid and retina occurs (concussion type of injury). The sclera remains intact. Vitreous hemorrhage may occur. The process usually ends up with a white fibrous scar and/or retinal pigmentary alterations which are often the final findings in this situation. The location is mostly at the site adjacent to the trajectory combined with part of the macula. 


\section{Case Report}

A 43-year-old male was admitted to the Department of Ophthalmology at Ziv Medical Center, Safed, Israel, due to a shrapnel injury to his right eye.

Upon admission, his visual acuity was light perception in his right eye and 6/12 in his left eye. Intraocular pressure was normal in both eyes. The right eye showed conjunctival and ciliary injection and 2 superficial minor small shrapnel were removed from the right cornea; apart from that, there was no pathology. The anterior chamber depth was normal with minimal flare. The pupil was round and regular with no relative afferent pupillary defect, and the lens was clear and in its normal position. Medium density vitreous hemorrhage was present obscuring the retina. A 4-mm-long entry wound was seen in the upper lid about $3 \mathrm{~mm}$ from the lateral canthus.

The left eye and the rest of the physical examination were entirely normal. On the computed tomography scan, shrapnel in the orbit's apex above the optic nerve in the right eye was demonstrated. Ocular ultrasonography demonstrated an elevation in the lower retina and high reflection in vitreous gel. The patient was treated with systemic and local antibiotics and steroids. Four days following his admission, the patient had a posterior pars plana vitrectomy conducted. During the procedure, an ischemic injury of the retina and choroidal tears were observed (Fig. 1, Fig. 2).

Upon discharge, the patient's visual acuity in the right eye was $1 / 120$, the vitreous was clear, and the retina showed a large fibrogliotic lesion associated with hyperpigmentation, few choroidal ruptures, and choroidal hemorrhages in a process of reabsorption.

\section{Discussion}

Retinitis sclopetaria is a relatively rare condition following a blunt trauma to the sclera due to trauma. It is a full-thickness disruption of the choroid and retina secondary to forces that are generated by the deceleration of a high-velocity projectile near the sclera, without penetrating the sclera and the eyeball. It is believed to result from shock waves that rupture the choroid and retina, but leave the sclera intact. Vitreous hemorrhages are common. Usually the lesions heal with a white fibrous scar and retinal pigment epithelium changes that are typically located in the peripheral retina.

The prognosis for visual acuity is poor. In case series of 13 patients, the final visual acuity ranged from no light perception to 20/1,200 with only very mild improvement in the course following the original trauma but only 1 of the patients developed retinal detachment in the injured eye [2]. The authors suggested that retinal detachment does not usually occur due to spontaneous retinopexy and scar formation. Most of the patients in this study were young males - the typical population that suffers from ocular trauma.

The reason for chorioretinitis sclopetaria is a high-velocity trauma usually due to gunshot or other military injuries. In one case report, the authors described a bilateral chorioretinitis sclopetaria due to gunshot trauma which emphasizes the need of a thorough examination of both eyes in cases of trauma [3]. In this study, the authors described the optical coherence tomography findings as bilateral full-thickness hyperreflectivity consistent with the chorioretinal disruption.

A trauma that causes chorioretinitis sclopetaria may cause other orbital damage. MacKenzie et al. [4] described a case of a patient who in addition to chorioretinitis sclopetaria suffered from a complete traumatic transection of the lateral rectus at the equator of the 
globe. In another case report, the authors described a concomitant optic nerve transection combined with chorioretinitis sclopetaria [5].

Chorioretinitis sclopetaria is a rare condition caused by a high-velocity trauma not penetrating the sclera and eyeball. Usually one of the findings is vitreous hemorrhage sometimes requiring posterior vitrectomy - as in our case - but retinal detachment only seldom occurs. Despite all efforts and treatments, visual prognosis is poor.

\section{Statement of Ethics}

The subject of this case report has given his informed consent. This study protocol has been approved by the Ziv medical center committee on human research.

\section{Disclosure Statement}

No funding was received for this research. The authors declare no conflict of interest.

\section{References}

1 Richards RD, West CE, Meisels AA: Chorioretinitis sclopetaria. Am J Ophthalmol 1968;66.5:852-860.

12 Ahmadabadi MN, Karkhaneh R, Roohipoor R, Tabatabai A, Alimardani A: Clinical presentation and outcome of chorioretinitis sclopetaria: a case series study. Injury 2010;41:82-85.

-3 Rayess N, Rahimy E, Ho AC: Spectral-domain optical coherence tomography features of bilateral chorioretinitis sclopetaria. Ophthalmic Surg Lasers Imaging Retina 2015;46:253-255.

4 MacKenzie K, Verity D, Ali N: Traumatic transection of the lateral rectus muscle with chorioretinitis sclopetaria. Strabismus 2015;23:36-38.

5 Mohammadpour M, Soheilian M: Concomitant optic nerve transection and chorioretinitis sclopetaria. BMC Ophthalmol 2005;5:29.

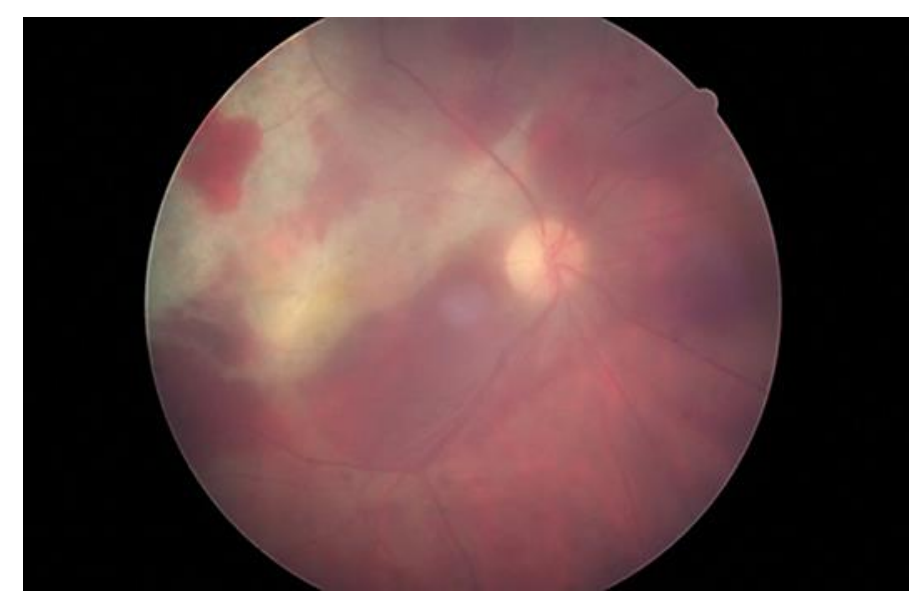

Fig. 1. Massive chorioretinitis, hemorrhages, and exudates. 

www.karger.com/cop

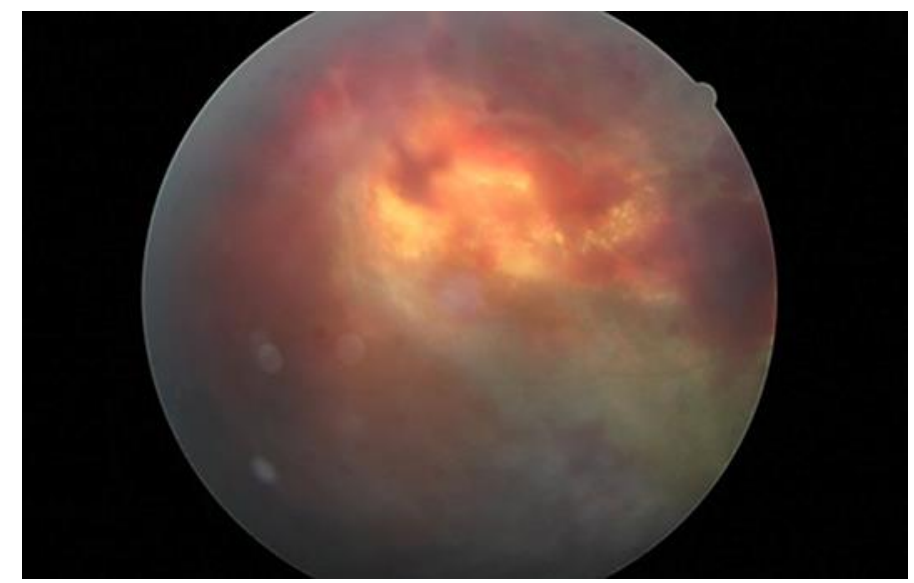

Fig. 2. Sclopetaria in the peripheral retina. 